FZMw Jg. 6 (2003) S. 208-215

\title{
Eva Rieger: Minna und Richard Wagner. Stationen einer Liebe. Düsseldorf und Zürich: Artemis \& Winkler 2003
}

\author{
Rezension von Melanie Unseld
}

$-1-$

Die Literatur über Richard Wagner füllt Dutzende von Buchregalmetern, jeder Winkel seines Lebens und Schaffens scheint ausgeleuchtet. Auch über seine Beziehungen zu Frauen wurde bereits viel geschrieben, dokumentiert und kommentiert. Warum also ein weiteres Buch über Wagner? Weil es tatsächlich eine wesentliche Lücke zu schließen gilt, eine Lücke, die erstaunlicherweise bisher niemandem aufgefallen zu sein scheint: Minna Wagner, Richards erste Ehefrau, erschien bislang allenfalls als Marginalie des Wagner-Schrifttums.

$$
-2-
$$

Das Schicksal, ihr Leben im Schatten des berühmten Ehemannes zu führen und damit von der Nachwelt bestenfalls als historische Randnotiz wahrgenommen zu werden, teilt Minna Wagner freilich mit vielen Frauen. Im Falle Wagners muss man jedoch von anderen Prämissen ausgehen: Frauen um Wagner zählen keineswegs zu jenen Vergessenen, die Wagner-Literatur wäre ohne Mathilde Wesendonck oder gar ohne Cosima Wagner undenkbar. Dass Minna Wagner bis dato derart wenig Beachtung geschenkt wurde, muss demnach andere Ursachen haben. Und mit der Suche nach diesen gerät man sogleich auch ins Zentrum der Angelegenheit: Denn in Minna Wagners Fall trug der Ehemann selbst erheblich dazu bei, den Mantel der scheinbaren historischen Irrelevanz über sie zu breiten. Durchaus doppeldeutig könnte man hier von einem Verdrängungsprozess sprechen, mit dem Wagner seine erste Ehefrau aus dem Kreis seines Lebens und seiner Selbstinszenierung als Künstler in die Unscheinbarkeit entließ. Dabei tilgte er geflissentlich Quellenmaterial, das auf eine andere Sichtweise hätte hindeuten können: Einen Großteil des Briefwechsels zwischen ihm und Minna vernichtete er, und wenn es je ein Doppelporträt der Eheleute gegeben hat (vergleichbar jenem bedeutsam inszenierten mit Cosima, auf dem sie vor ihm sitzt und bewundernd zu ihm aufschaut), so ist es nicht überliefert. 
Auf dem Titelbild der von Eva Rieger nun vorgelegten Doppelbiographie Minna und Richard Wagner. Stationen einer Liebe erscheinen denn auch die beiden Figuren seltsam verbindungslos montiert, sie als rotwangige Schönheit, er schattenhaft, gleichsam unterbelichtet im Hintergrund. Aber im Gegensatz zu diesem ersten visuellen Eindruck gelingt es der Autorin, das Schlaglicht auf b e i d e Personen zu werfen, beide zu Wort kommen zu lassen, beide in ihrer gegenseitigen Abhängigkeit darzustellen - und so letztlich auch den Konflikt nachvollziehbar zu machen, der nicht nur zur Entfremdung der Eheleute führte, sondern auch zu jener historischen Schieflage, die Minna Wagner fast vollständig von der historiographischen Bildfläche verschwinden ließ.

Um die erstaunliche Leistung des Buches zu ermessen, muss man sich die Ausgangslage dieser Doppelbiographie vergegenwärtigen: Nicht nur, dass hier eine gut dokumentierte, vielfach kommentierte Lebensgeschichte mit einer nur ausschnitthaft überlieferten gleichberechtigt ineinanderverwoben wurde. Rieger hatte sich vor allem auch mit den (selbst-)inszenatorischen Fähigkeiten Richard Wagners auseinanderzusetzen und dabei behutsam das Wunschbild von der historischen Wahrscheinlichkeit zu trennen. Richard Wagners Blick auf sein eigenes Leben war stark von Wunschvorstellungen, Idealisierungen und utopischen Zukunftsplänen geprägt - eine Tatsache, die seine alltagsgeprüfte Frau zeitlebens kritisierte, unter der sie litt und die zugleich einem heutigen Betrachter einen besonders kritischen Blick auf die Quellen abnötigt. Was hat es beispielsweise zu bedeuten, wenn Wagner in einem Brief an seine Frau wortreich und glaubhaft seiner Sehnsucht nach ihr Ausdruck verleiht und zugleich an den Freund Franz Liszt schreibt, dass ihm seine Ehefrau eher lästig denn lieb sei?

$$
-5-
$$

Dass die Ehe in späteren Jahren problematisch, ja zerrüttet war, hat sich fest ins historische Gedächtnis eingeprägt, weniger jedoch, daß dieser Zeit ein gemeinsamer Weg vorausgegangen war, ein Weg, der den Künstler Richard Wagner tief prägte und während dem ein Großteil seiner Werke entstand oder konzipiert wurde.

$$
-6-
$$

Minna Planer verfolgte - aus ärmlichen Verhältnissen kommend - eine Karriere als Schauspielerin, die landesweit Beachtung fand. Rieger flicht einige kulturhistorische Anmerkungen ein, so etwa Details zum Beruf der Schauspielerin, was nicht nur zu einem facettenreicheren Gesamtbild beiträgt, sondern sich später auch für ein tieferes Verständnis Minna Wagners als unerlässlich erweist: Da den Schauspielerinnen 
vorgeschrieben war, für ihre Theaterroben und Kostüme selbst aufzukommen, war der durchaus einträgliche Verkauf von Minnas Bühnengarderobe nicht nur ein finanzielles Opfer, sondern zugleich auch ein emotionales: Minna besiegelte damit ihren endgültigen Abschied von der Bühne.

$$
-7-
$$

Als Minna Planer den jungen Richard Wagner kennen lernte, zögerte sie zunächst, ihre Bühnenlaufbahn aufzugeben, da er weder eine Anstellung noch Aussicht auf Erfolg als Komponist hatte. Die Armut ihrer Kindheit und die bittere Erfahrung, bereits mit 16 Jahren Mutter einer unehelichen Tochter geworden zu sein, hatten sie gelehrt, pragmatisch zu denken und zu handeln, und so widerstand sie zunächst ihrer eigenen Neigung und den eloquenten Bittbriefen Wagners. Minna Wagner resümierte später: "Was warst Du denn als ich Dich heirathete? Du warst ein armer, verlassner, unbekannter, unangestellter Musikdirector, und was standen mir damals für Aussichten bevor! [...] ich [tat] ja Alles aus Liebe, sogar meine Selbständigkeit die ich so hoch hielt, gab ich freudig auf, um Dir ganz angehören zu können." (S. 56)

$$
-8-
$$

Doch Wagner verstand sich schon damals auf die hohe Kunst des 'Bettelbrief'Schreibens, die er im Verlauf der kommenden Jahre immer mehr verfeinerte (nicht nur gegenüber Minna, sondern auch - in meist finanziellen Angelegenheiten - gegenüber seinen Freunden und Gönnern). Dass Rieger aus diesen Briefen ausführlich zitiert, lässt den Leser ungemein lebendig teilhaben an Minna Planers schwieriger Situation: auf der einen Seite die Notwendigkeit, als Schauspielerin den Lebensunterhalt für sich, ihre Tochter und ihre Eltern zu bestreiten, auf der anderen Seite von jenem stürmisch liebenden, vier Jahre jüngeren, als Kapellmeister wie als Komponist freilich bislang noch wenig erfolgversprechenden Mann derart umworben zu werden.

$$
-9-
$$

Neben der gegenseitigen Liebe war es vor allem auch Minnas Glaube an Richards zukünftigen Erfolg, der die junge Frau hoffen ließ: "Sie hielt jedoch aus Liebe zu ihm und - was noch wichtiger war - sie glaubte an ihn", kommentiert Rieger (S. 56). Doch der Weg zum Erfolg sollte für beide noch langwierig und äußerst steinig werden. Minna ging ihn unter vielen Entbehrungen und zuweilen auch unter Einsatz ihres Lebens mit (die späteren Erfolge und den Ruhm teilte Wagner dann bekanntlich mit anderen Frauen). 
Die Ehe war jedoch von Anfang an konfliktreich: Wagner war chronisch eifersüchtig, launisch und ohne die beständige finanzielle Unterstützung durch Freunde und Gönner nicht in der Lage, den Lebensunterhalt für sich und seine Frau zu bestreiten. Gerade letzter Punkt führte immer wieder zu Diskussionen zwischen den Eheleuten, da Minna nicht nur ihre ökonomische Selbständigkeit für ihn aufgegeben hatte, sondern sich aus Schamgefühl beharrlich weigerte, Geld von Freunden anzunehmen. Daran, dass die meist unrealistischen, dabei aber hochfliegenden finanziellen Pläne Richards in der Regel scheiterten, musste Minna sich bald gewöhnen (was auch dazu führte, dass sie seinen Versprechungen immer weniger Glauben schenkte), dass er aber dennoch seinen Hang zum luxuriösen Leben nicht aufzugeben bereit war, stellte sie, die sich um die alltäglichen Dinge zu kümmern hatte, vor massive Probleme. Sie warf ihm seine Unfähigkeit vor, finanziell für eine Familie zu sorgen, während er sie kleinlich nannte, da sie beständig die stets akuten Geldsorgen thematisierte. Doch warum geht Rieger derart ausführlich auf die finanziellen Schwierigkeiten ein? Warum beschreibt sie ostentativ die ewigen Geldnöte? Gerade am leidigen Thema 'Geld' kann Rieger einen Grundzug der schwierigen Beziehung zwischen Minna und Richard Wagner aufzeigen: Richard war hochgradig von Minna abhängig, nicht nur emotional (was Rieger ebenfalls treffend zu schildern weiß), sondern auch von ihrem Realitätssinn, ihrem praktischen Engagement, ihrer Fähigkeit, mit Armut umzugehen - kurz: von ihrer Fähigkeit, den schwierigen Alltag (gerade auch in der bitteren Not der Pariser Jahre), bei den unzähligen Umzügen und während des Exils zu meistern - , ihm überhaupt die notwendigen Freiräume für sein Schaffen zu ermöglichen. Dies hätten ihm, so betont Rieger zu Recht, weder Mathilde Wesendonck noch Cosima von Bülow, beide aus wohlgeordneten, finanziell unabhängigen Verhältnissen stammend, geben können: "In diesen Jahren hätte ihm eine ekstatisch Liebende, wie sie später Jessie Laussot, Mathilde Wesendonck und Cosima von Bülow verkörperten, kaum etwas genützt. Er brauchte eine Frau mit Herz, Verstand und viel Takt, die ihm in der strengsten Entbehrung beistand, und Minna erfüllte diese Rolle." (S. 18)

$$
-11-
$$

Wagner war diese Abhängigkeit durchaus zuweilen bewusst. So schreibt er ihr in einem Brief vom Sommer 1859: 
"...denn ich muß mir ja sagen, daß für so viele Beschwerden und Kümmernisse des Lebens, die Dir durch Deine Vereinigung mit mir bereitet worden sind, die Gaben meiner Kunst die einzigen mir möglichen Entschädigungen sind: nimm die erhebenden Eindrücke [...] so auf, und bedenke, daß, wer das leistet, was ich leiste, hauptsächlich nur mit diesen Leistungen andren auch ihre Opfern lohnen kann. Liebe daher auch meinen Lohengrin; er gehört zu dem, was ich Dir einzig bieten kann!" (S. 402).

Und Minna nahm tatsächlich die frühen Werke nicht nur als Zeichen von Wagners eminentem Können (an das sie stets geglaubt hatte), sondern auch als Zeichen einer geglückten, da produktiven Partnerschaft.

$$
-12-
$$

Wagner freilich scheint seine Abhängigkeit von seiner Ehefrau häufig unangenehm, wenn nicht gar peinlich gewesen zu sein. Und es ist typisch für Wagners sprunghaften Charakter, dass er diese Abhängigkeit durchaus auch verleugnete. Dazu gehörte, dass er vor allem nach außen hin bald begann, die Ehe negativ darzustellen. Seiner Behauptung etwa, Minna habe seine Kunst nicht wirklich verstehen können, kann man so uneingeschränkt nicht zustimmen: Sie verfolgte mit großem Interesse den Fortgang jeder Komposition, unterstützte Richard im Kompositionsprozess, indem sie ihm den notwendigen Freiraum schaffte, und setzte sich für Aufführungen seiner Werke in Deutschland zu Zeiten des Schweizer Exils ein. Freilich: den Tristan konnte sie kaum gutheißen, spiegelte sich in ihm doch die Liebesaffäre mit Mathilde Wesendonck wider, die 1858 zum Eklat und zum Auszug aus dem Zürcher 'Asyl' führte. Und auch dem Ring stand sie skeptisch gegenüber, schrieb Wagner ihr doch unverblümt, ihre Person stimme mit der wenig schmeichelhaften Figur der Fricka "gar nicht übel zusammen" (S. 231).

$$
-13-
$$

Die Widersprüche zwischen dem, was Richard Wagner der Außen- und Nachwelt über seine erste Ehefrau mitteilte, und dem, was Eva Rieger aus Archiven, Briefsammlungen und anderen Quellen zusammentragen konnte, sind zahlreich. In seiner Autobiographie etwa spricht Wagner ihr selbst ihre schauspielerischen Qualitäten ab ("Talent für das Theater besa $\beta$ sie [...] nicht"), obgleich zeitgenössische Rezensionen und auch die Anzahl der Engagements und Engagementangebote, die die Schauspielerin Minna Planer vor ihrer Verheiratung erhielt, eine deutlich andere Sprache sprechen. 
Rieger stellt die Quellen nebeneinander, zitiert ausführlich - und nimmt sich als Kommentatorin dabei auffallend zurück. So manche Ungerechtigkeit, die Minna Wagner von ihrem Mann erfahren musste, wird geschildert, aber nicht bewertet. Rieger überlässt damit souverän das Terrain der emphatischen Reden und Gegenreden den Hauptakteuren, Richard und Minna Wagner, und das der Wertung dem lesenden Publikum. Diese Haltung mag anfangs irritieren, zumal Rieger auch da nicht den kürzenden Rotstift ansetzt, wo jeder pflichtbewusste Lektor der weitschweifigen Eloquenz Richards Einhalt geboten hätte. Schnell aber wird deutlich, dass nur so auch die Reaktionen Minnas nachvollziehbar werden: warum sie immer wieder seinen Überredungskünsten erlag, zu ihm zurückkam, mit ihm eine lebensbedrohliche (und eine Fehlgeburt verursachende) Flucht durchstand, wieder und wieder einen neuen Hausstand aufbaute. Von Richards Überredungskünsten zu hören, ist eine Sache, sie selbst zu lesen, ihnen wahrhaft 'ausgesetzt' zu sein, eine andere.

$$
-15-
$$

Die zurückhaltende Position Riegers überzeugt vor allem dort, wo der Ehekonflikt eskaliert. Rieger bereitet beide Positionen auf und überlässt auch hier dem Leser die Parteinahme. Dass sie dabei tendenziell mehr über Minna Wagner klären und erläutern muss, liegt in der Natur der Sache: Wagners publizistische Plattform war und ist ungleich größer.

$$
-16-
$$

Rieger trägt behutsam auch jene Schichten ab, die die Historiographie über die Person Minna Wagners gelegt hat. Die Wagner-Biographik ging, so Rieger, bislang recht wahllos mit der Figur Minnas um, mal ist sie die "Herzensbrecherin", die "Zurückhaltung und Koketterie im Typ" vereint (Martin Gregor-Dellin), mal schlüpft sie in die Rolle der "leichtlebigen Ehebrecherin" (Joachim Köhler). Vor allem scheint die 'Schuldfrage' des Zürcher Eklats eindeutig geklärt: "Die Wagnerbiographik hat beharrlich die Legende kolportiert, wonach Minna die alleinige Schuldige an dem Eklat war." (S. 293)

$$
-17-
$$

Mit diesen Zuschreibungen, die nach den hier zusammengetragenen Quellen unterschiedlicher Provenienz nicht haltbar sind, räumt Rieger auf. Gerade hier erweist sich, wie gewinnbringend ihre Methode ist, die Texte selbst sprechen zu lassen, unterschiedliche Textquellen einander gegenüberzustellen, um aus den Übereinstimmungen und Widersprüchen, aus den unterschiedlichen Schattierungen und Nuancen zu einer historischen Wahrscheinlichkeit zu gelangen. Denn gerade Wagners Autobiographie ist 
ein treffliches Beispiel, wie behutsam die Geschichtsschreibung mit Quellenmaterial umzugehen hat. Im Falle von Mein Leben muß man sich die Entstehungssituation des Buches vor Augen halten, um zu erkennen, wer hier wen wie betrachtete: Richard diktierte seine Erinnerungen seiner Noch-nicht-Ehefrau Cosima von Bülow in die Feder, was im Hinblick auf die Gerade-noch-Ehefrau Minna selbstverständlich zu ganz eigenen Interpretationen führen mußte: "Richard stand vor dem Problem," so erläutert Rieger, "seiner zweiten Frau Cosima und einer eingeweihten Leserschaft zu erklären, warum er sich von Minna trennen mußte, und zugleich aber herauszustellen, was ihn bewog, gegen den Willen seiner Verwandten und über alle Hindernisse hinweg Minna geheiratet zu haben." (S. 19)

$$
-18-
$$

Den mühsamen - aber erhellenden - Weg, hinter die Masken der Selbst- und Fremdinszenierungen zu schauen, nimmt Rieger in ihrer Doppelbiographie erstmals auf sich. "Es gilt [...], die Ereignisse aus ihrem Entstehen heraus zu deuten, alle Quellen mit Vorsicht zu behandeln und erst in der Häufung von Briefen, Erinnerungen, zeitgenössischen Äußerungen und historischen Fakten nach dem zu suchen, was der Wirklichkeit am nächsten kommt." (S. 21) Rieger ist dabei umsichtig genug, auch i h r e Sicht auf das Ehepaar Wagner allenfalls als e i n e Lesart zu verstehen:

"Die Neigung des Biographen oder der Biographin, die zu beschreibende Person in Schutz zu nehmen und notfalls zu idealisieren, ist oft zu Recht kritisiert worden. Ganz frei ist man von Solidarisierungseffekten - trotz aller Bemühungen - freilich nicht. Die Verfasserin bekennt, sich leichter in Minnas als in Richards Situation hineinversetzen zu können, doch wäre niemandem gedient, wollte man bei aller Kritik Richards ihn mit Häme überziehen und als menschlich charakterlos darstellen, wie es oft genug geschehen ist." (S. 21)

Und hierin ist das Buch schlichtweg überzeugend: Rieger gelingt es, in einem derart heiklen Beziehungsgeflecht sich einer wertenden Parteinahme zu enthalten. $\mathrm{Zu}$ Hilfe nimmt sie dabei immer wieder den Blick auf die Geschlechtergeschichte des bürgerlichen 19. Jahrhunderts, das den Individuen stark ausgeprägte Rollen - Mann und Frau, Ehebrecher und betrogene Gattin, Künstler und Schauspielerin - zugewiesen hatte. Indem Rieger dies als Hintergrundfolie stets bereit hält, werden nicht nur so manche Entscheidungen (und Nicht-Entscheidungen) verständlich, sondern auch jener Schritt, 
den Richard Wagner schließlich in der Sublimierung alltäglicher Lebens- und Liebeskonstellationen in seinen Werken tat. Dass letzteres in Riegers Buch nur noch am Rande anklingt, kann man dem über 400seitigen Werk nicht anlasten, es ist vielmehr ein Ausblick auf das, was auf der Grundlage von Riegers Doppelbiographie nun zu erhoffen ist. 